\title{
Un cas de prise en charge inadaptée d'une fracture sous condylienne bilatérale traitée par ostéosynthèse seule. \\ Vandeputte $\mathrm{T}^{1}$, Maladiere $\mathrm{E}^{2}$, Fauroux $\mathrm{MA}^{3}$, Torres $\mathrm{JH}^{3}$ \\ 1. Centre de soins et de Recherche Dentaires de Montpellier - CHU Montpellier \\ 2. Service CMF - CHU Perpignan \\ 3. Service d'Odontologie de Montpellier - Université Montpellier I : EA4203
}

\section{Introduction}

Les fractures mandibulaires représentent une des urgences traumatologiques les plus fréquentes en chirurgie maxillo-faciale. En fonction du type de fracture et de la localisation, le traitement est chirurgical ou pas. Le principe du traitement chirurgical consiste à ostéosynthéser les fragments en position d'articulé de référence afin qu'il ne persiste pas de trouble occlusal résiduel(1).

\section{Observation}

Un homme de 35 ans a consulté 1 mois après une fracture bicondylienne suite à un malaise survenu lors d'un séjour à l'étranger. II présentait une fracture sous-condylienne bilatérale : basse à gauche, qui avait fait l'objet d'une ostéosynthèse, et haute à droite, qui n'avait pas été chirurgicalement. Le patient n'avait bénéficié d'aucune rééducation par kinésithérapie post-opératoire. A de son retour en France, il a consulté au service de Chirurgie Maxillo-Faciale pour un trouble occlusal majeur apparu secondairement l'empêchant de s'alimenter correctement. On retrouvait à l'examen clinique une béance antérieure, un contact molaire prématuré bilatéral et une amplitude d'ouverture buccale à $3 \mathrm{~cm}$. A la radiologie panoramique dentaire et à l'examen tomodensitométrique ont été constatés une réduction incomplète des foyers de fracture, un trait de fracture symphysaire non déplacé, ainsi qu'une plaque d'ostéosynthèse au niveau sous-condylien bas gauche. Des empreintes ont été prises afin de confirmer l'absence de troubles occlusaux antérieurs. La question d'une reprise chirurgicale s'est alors posée, avec deux options : celle d'une nouvelle réduction avec ostéosynthèse à court terme, dans les jours suivants pour ne pas laisser l'os consolider davantage ou celle d'une chirurgie orthognathique à distance, après au moins un an. Finalement, un simple traitement orthopédique a été entrepris. Des arcs de Dautrey ont été mis en place afin d'obtenir un blocage sur élastique, et une rééducation par kinésithérapie a été débutée. Deux mois après le début de la rééducation, des résultats satisfaisants ont été constatés avec une occlusion correcte, ce qui rendait inutile le recours à une chirurgie.

\section{Discussion}

A la vue des résultats, l'indication première d'ostéosynthèse doit être discutée dans ce type de fractures. En effet, une rééducation isolée aurait pu suffire, d'autant que l'articulé dentaire avait été conservé initialement. De plus, la chirurgie des fractures sous-condyliennes devrait être réservée à des chirurgiens entrainés car elle est source de complications, notamment de paralysies faciales iatrogènes.

\section{Conclusion}

Ce cas illustre non seulement l'importance de la rééducation par un kinésithérapeute dans les fractures souscondyliennes mais aussi celle des examens cliniques et radiographiques dans les choix thérapeutiques.

younghurdler@hotmail.fr

Références

(1)Trost $\mathrm{O}$ et Peron JM. Rev Stomatol Chir Maxillofac Chir Orale 2013; 114 : 341-348

(C) The authors, published by EDP Sciences. This is an Open Access article distributed under the terms of the Creative Commons Attribution License 4.0 (http://creativecommons.org/licenses/by/4.0/). 